\title{
The comparison of $0.05 \%$ sodium fluoride and $0.2 \%$ chlorhexidine usage and aquadest to the plaque index on fixed orthodontic patients
}

\author{
Veronica Vera D. Wiraja, Tono S. Hambali, Soertini E. Lambri \\ Department of Orthodontic Faculty of Dentistry, Universitas Padjadjaran
}

\begin{abstract}
\end{abstract}
Fixed orthodontic appliance will cause changes in microbial flora the oral cavity and food debris accumulation, and will be formed especially around the gingival sulcus. Plaque control using chemical means can be done by using mouth rinse. This research compared the influence of $0.05 \%$ sodium fluoride mouth rinse with aquadest, and $0.2 \%$ chlorhexidine to the plaque index in fixed orthodontic patients. A double blind and cross over clinical assessment were applied using a sample size of 16 male fixed orthodontic patients with the age above 21 years. $0.05 \%$ sodium fluoride, $0.2 \%$ chlorhexidine mouth rinse was given to all patients as a positive control, and aquadest as a negative control. Plaque index was then measured after 24 hours without tooth brushing, after using the mouth rinse and a week after using the mouth rinse with tooth brushing. The results showed that the use of $0.05 \%$ sodium fluoride mouth rinse reduced plaque index more significantly compared to $0.2 \%$ chlorhexidine. The mechanical plaque control by tooth brushing is still the most influential mean to reduce plaque index in fixed orthodontic patients. Mouth rinse is just an additional mean to reduce plaque.

Key words: Sodim fluoride, chlorhexidine, aquadest, plaque index, fixed orthodontics

\section{INTRODUCTION}

Orthodontic treatment rises because there is a constant need to fix the patient's dentofacial problems. ${ }^{1}$ Orthodontic treatments especially fixed orthodontics will increase the risk of plaque forming Therefore, during the treatment the tooth, gingival and periodontal tissue health condition will require some attention. The use of fixed orthodontic appliances such as band, brackets, ligature braces, and elastic rubbers inside the patient's mouth will trigger the changes on oral cavity environment which will cause increasing of the microbial flora concentration, especially acid producing bacteria and the accumulation of food to form plaque. ${ }^{2,3}$ The plaque type on fixed orthodontic patients is more cariogenic because of the increase of carbohydrate concentration per milligram plaque formed, caused by the increase of lactobacilli population.

There are two ways in preventing plaque accumulation; mechanically (by tooth brushing) and chemically. The prevention of plaque accumulation chemically to prevent caries forming and periodontal tissue damage on fixed orthodontic patients was by using a mouth rinse. ${ }^{4}$ Mouth rinse was proven to be effective in many researches on plaque control and prevention of gingivitis, using 
$0.2 \%$ and $0.12 \%$ chlorhexidine gluconate, but in the long-term usage of chlorhexidine gluconate, it has a side effect of loosing the taste sensation on tongue and coloring the teeth and tongue in the form of black hairy tongue. ${ }^{1,5}$ Because of these reasons, the usage of chlorhexidine mouth rinse on fixed orthodontic patients was limited. ${ }^{1,3,6}$ According to the writer's observation, in Indonesia chlorhexidine mouth rinse is only available commercially in $0.2 \%$ concentration (Minosep $®$ ).

Other mouth rinse which was recommended for daily use during orthodontic treatments was fluoride mouth wash with. ${ }^{6}$ Fluoride in the form of mouth rinse will reduce enamel decalcification, the possibility of plaque forming, and gingivitis. ${ }^{7}$ Fluoride has the ability to reduce the supragingival plaque by reducing the Streptococcus mutans proportion, with minimal fluorosis side-effect. ${ }^{8}$ Commercially, there are various mouth fluoride rinse preparation with in several concentration. According to the writer's observation, fluoride mouth rinses available in Indonesia are mostly $0.05 \%$ sodium fluoride, which are Pepsodent Mouthwash ${ }^{\circledR}(0.05 \%$ sodium fluoride), Oral B Mouthrinse ${ }^{\circledR}(0.053 \%$ cethylpyridinium chloride dan $0.05 \%$ sodium fluoride), and Listerine Teeth and Gum ${ }^{\circledR}$ (Essential oil dan $0.02 \%$ sodium fluoride). Several literatures stated that sodium fluoride in $0.05 \%$ concentration ( $225 \mathrm{ppm}$ ) was recommended for daily use during orthodontic treatments..$^{9-12}$

Meanwhile, the usage of water as chemical substance in plaque control has no benefit. Newman was conducting a research using water as an irrigation source, and he stated that water can be used as an irrigation after tooth brushing, but it does not reduce the plaque. Chaves' research ${ }^{3}$ using WaterPik device showed that water cannot reduce the forming of plaque chemically.

According to those facts, the writer compared the influence of $0.05 \%$ sodium fluoride mouth rinse commercially available in Indonesia, with aquadest (purified water - $\mathrm{H} 2 \mathrm{O}$-subsitute as normal water for rinsing the mouth) as a negative control, and $0.2 \%$ chlorhexidine as a positive control towards plaque index in fixed orthodontic patients. The writer wished to see whether $0.05 \%$ sodium fluoride commercially available in Indonesia has an effect towards plaque index on fixed orthodontics patients.

\section{METHOD}

Samples in this experiment was fixed orthodontic patients who undergone treatments in Orthodontics Specialist Clinic of Faculty of Dentistry Universitas Padjadjaran; male, didn't have any systemic disease, currently not taking any antibiotics in the last two months, and never suffer periodontal disease and have an age above 21 years. By using male samples above 21 years old, hormonal effects were diminished and the risk of fluorosis was avoided. The mouth rinse used was $0.05 \%$ sodium fluoride mouth rinse (Pepsodent Antiplaque Mouthwash $®), \quad 0.2 \% \quad$ chlorhexidine (Minosep ${ }^{\circledR}$ ), and aquadest. In addition, disclosing solution (GUM from Sunstar Inc, Chicago, USA $₫$ ) was used as a plaque marker, toothpaste (Pepsodent $®$ ) hand piece with the brush, basic tools (mirror, explorer, tweezer, and excavator) and periodontic probe.

The amount of samples fit the criteria was 16 male patients out of 41 population with a confidence limit of (d́) 95\% ( $p=0.05)$. This research was only to count the plaque index on the labial area on maxilla teeth using brackets, to facilitate plaque index count correctly. All of the mouth rinses used was transferred in closed bottles with the same cap labels [to prevent the sight of different colors of mouth wash used by samples and researchers] The amount of the mouth wash used was measured the same as the factory instruction, which was $15 \mathrm{ml}$ for $0.05 \%$ sodium fluoride mouth rinse and $10 \mathrm{ml}$ for $0.2 \%$ chlorhexidine mouth wash, and $15 \mathrm{ml}$ for the the aquadest. Samples received fostering about the correct tooth brushing and mouth rinsing technique for fixed orthodontics patients. All samples received a new orthodontic toothbrush (Oral $B \otimes$ ) and the same toothpaste (Pepsodent $\AA$ ).

The cross over design was used to test the effectiveness of plaque index decrease using the $0.05 \%$ sodium fluoride, and a mouth wash with $0.2 \%$ chlorhexidine as the positive control on each sample. Aquadest was used as a negative control on the third week after the wash out period for 1 week. The wash out period for 1 week on the second week of experiment was used to diminish the effect of mouth wash previously used. The double blind technique used in this research was 


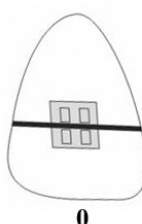

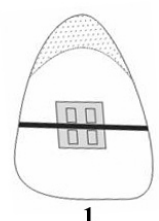

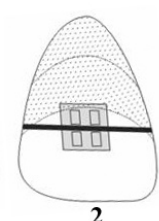

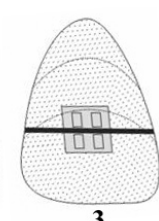

Figure 1. Modification plaque index from Simplified Oral Hygiene Index (OHI-S) Greene and Vermillion. ${ }^{14}$

$0=$ no plaque $; 1=$ Plaque coverage one third of tooth surface; $2=$ plaque coverage is two third of tooth surface; $3=$ plaque covers more than two third of tooth surface

meant to avoid the perception of researchers and samples towards a certain brand of mouth wash, so that the trained and calibrated researcher in Periodontology Department and the samples did not know about the mouth wash used by the samples. Samples were exposed to prophylaxis beforehand, and then they were instructed not to brush their teeth for 24 hours. The forming plaque after 24 hours was the plaque biofilm which was dominated by plaque forming bacteria, especially Streptococcus. Because of the populating bacteria, after 24 hours plaque will start to attach to the tooth surface. ${ }^{14}$

After 24 hour of non tooth-brushing, the plaque indexes on the samples were measured, only on the maxillary labial teeth wearing brackets. The plaque index used was modification of plaque index from Simplified Oral Hygiene Index (OHI-S) Greene and Vermillion. Simplified Oral Hygiene Index (OHI-S) Greene and Vermillion is one of the index counts for areas. ${ }^{14}$

After the measurement of early plaque index, samples were then divided into 2 groups. On the first group, the samples were instructed to rinse their mouth by using $15 \mathrm{ml} 0.05 \%$ fluoride mouth rinse (according to the manufacturer's instruction), then the plaque index was measured. After the plaque index was measured, samples were instructed to brush their teeth and use 15 $\mathrm{ml} 0.05 \%$ sodium fluoride mouthwash twice a day for 1 week (treatment I). A week later, the plaque index was re-measured. After undergoing a wash out period (not using the mouth rinse) for a week, samples received prophylaxis and were instructed not to brush their teeth for 24 hours.

After 24 hours on non tooth-brushing, the plaque index was measured again and samples were instructed to rinse their mouth by using 15 $\mathrm{ml}$ aquadest mouth rinse, and the plaque index was measured again. There after the samples were instructed to brush their teeth and use the $15 \mathrm{ml}$ measured aquadest mouth rinse, twice a day for one week, and the plaque index was measured again.

At the final stage of the research, the samples were given prophylaxis and were instructed not to brush their teeth for 24 hours. After 24 hours, the plaque index was measured and the samples were instructed to rinse their mouth by using $10 \mathrm{ml} 0.2 \%$ chlorhexidine (according to the manufacturer's instruction) then the plaque index was measured again. Samples were instructed to brush their teeth and use the $10 \mathrm{ml} 0.2 \%$ chlorhexidine mouth rinse, for twice a day for one week. One week later, the plaque index on the samples were measured again (treatment II). Meanwhile, by using the same research method, the second group was instructed to use $0.2 \%$ chlorhexidine mouth rinse (treatment II), then the aquadest, and the last one was $0.05 \%$ sodium fluoride mouth rinse (treatment I), each was used twice a day. The measurement of the two groups was compared in the tables below.

\section{RESULT}

The average plaque index on the use of three different mouth rinses in this research is described in the table below. IP A was the early plaque index after samples didn't brush their teeth for 24 hours, while IP B was the plaque index after patient's rinses using the mouth rinse without brushing their teeth, and IP P was the research plaque index taken after patients brush their teeth and rinse using the mouthwash for one week. Data obtained was then averaged, statistically measured and compared between their intra and intergroup. The results on Table 2 were the comparison on plaque index on each mouth wash (intragroup) on 3 different times. From the results on table 2 , the plaque index was converted into percentage (Table 3 ) to test the result of the research, because of the time frame difference between mouth wash in intragroups.

From the statistical results in Table 3 , it can be read as the intragroup statistical results, because from the difference on the research's time frame, $0.05 \%$ sodium fluoride mouth wash significantly decrease the plaque index in the test of the three plaque index. 
The comparison of sodium fluoride $0.05 \%$ and chlorhexidine $0.2 \%$ usage and aquadest (Veronica VD, et.al.)

\section{RESEARCH DIAGRAM}


Table 1. Results showing plaque index from 3 kinds of mouthwash $(n=16)$

\begin{tabular}{ccc}
\hline Mouthwash & Plaque index & Average(\%) \\
\hline $\begin{array}{cc}\text { Chlorhexidine } \\
\text { (CHX) }\end{array}$ & IPA & 2.47 \\
& IPB & 2.03 \\
IP P1 & 0.10 \\
Sodium Fluoride & IP A & 2.51 \\
$(\mathrm{NaF})$ & IP B & 2.10 \\
& IP P2 & 0.12 \\
Aquadest & IP A & 2.17 \\
$\left(\mathrm{H}_{2} \mathrm{O}\right)$ & IP B & 2.17 \\
& IP P3 & 0.63 \\
\hline
\end{tabular}

Table 2. Index change test according to time

\begin{tabular}{ccc}
\hline Mouthwash & Plaque index & Average(\%) \\
\hline $\begin{array}{c}\text { Chlorhexidine } \\
\text { (CHX) }\end{array}$ & IP A vs IP B & 0.44 \\
& IP A vs IP P1 & 2.38 \\
& IP B vs IP P1 & 1.94 \\
Sodium Fluoride & IP A vs IP B & 0.41 \\
(NaF) & IP A vs IP P2 & 2.39 \\
& IP B vs IP P2 & 1.98 \\
Aquadest & IP A vs IP B & 0.00 \\
( $\left.\mathrm{H}_{2} \mathrm{O}\right)$ & IP A vs IP P3 & 1.54 \\
& IP B vs IP P3 & 1.54 \\
\hline
\end{tabular}

Table 3. Plaque index change based on time (in \%)

\begin{tabular}{|c|c|c|c|c|c|c|c|}
\hline Mouthwash & Plaque index & Average(\%) & std & $\mathrm{n}$ & t Count & $\mathrm{t}$ table & Characteristic \\
\hline $\begin{array}{c}\text { Chlorhexidine } \\
\text { (CHX) }\end{array}$ & $\begin{array}{l}\text { IP A vs IP B } \\
\text { IP A vs IP P1 } \\
\text { IP B vs IP P1 }\end{array}$ & $\begin{array}{l}17.53 \\
96.11 \\
95.05\end{array}$ & $\begin{array}{c}12.429 \\
3.039 \\
3.863\end{array}$ & $\begin{array}{l}16 \\
16 \\
16\end{array}$ & $\begin{array}{c}5.64 \\
126.50 \\
98.41\end{array}$ & $\begin{array}{l}2.13 \\
2.13 \\
2.13\end{array}$ & $\begin{array}{l}\text { Sign } \\
\text { Sign } \\
\text { Sign }\end{array}$ \\
\hline $\begin{array}{l}\text { Sodium Fluoride } \\
\qquad(\mathrm{NaF})\end{array}$ & $\begin{array}{l}\text { IP A vs IP B } \\
\text { IP A vs IP P2 } \\
\text { IP B vs IP P2 }\end{array}$ & $\begin{array}{l}16.45 \\
95.22 \\
93.90\end{array}$ & $\begin{array}{l}14.361 \\
3.657 \\
4.968\end{array}$ & $\begin{array}{l}16 \\
16 \\
16\end{array}$ & $\begin{array}{c}4.58 \\
104.14 \\
75.60\end{array}$ & $\begin{array}{l}2.13 \\
2.13 \\
2.13\end{array}$ & $\begin{array}{l}\text { Sign } \\
\text { Sign } \\
\text { Sign }\end{array}$ \\
\hline $\begin{array}{l}\text { Aquadest } \\
\qquad\left(\mathrm{H}_{2} \mathrm{O}\right)\end{array}$ & $\begin{array}{l}\text { IP A vs IP B } \\
\text { IP A vs IP P3 } \\
\text { IP B vs IP P3 }\end{array}$ & $\begin{array}{c}0.00 \\
69.67 \\
69.67\end{array}$ & $\begin{array}{l}0.000 \\
9.515 \\
9.515\end{array}$ & $\begin{array}{l}16 \\
16 \\
16\end{array}$ & $\begin{array}{c}0.00 \\
29.29 \\
29.29\end{array}$ & $\begin{array}{l}2.13 \\
2.13 \\
2.13\end{array}$ & $\begin{array}{l}\text { NonSign } \\
\text { Sign } \\
\text { Sign }\end{array}$ \\
\hline
\end{tabular}

Note: IP A: preliminary plaque index after sample not brushing their teeth for 24 hours; IP B:Plaque index after samples rinses with the mouthwash without brushing their teeth; IP P:Research plaque index after the patient brush their teeth and rinse for a week; std: Deviation standard; n: Sample numbers; Sign: Statistically significant (t table<t count); Non Sign: Not significant statistically ( $\mathrm{t}$ table $>$ t count)

The mouth wash $0.05 \%$ sodium fluoride significantly decrease the early plaque index after 24 hours of not brushing the teeth (IP A) compared with the plaque index rinsing without brushing the teeth (IP B) by the average of $6.45 \%$. Meanwhile on IP A test with research plaque index after 1 week of using the mouthwash and brushing the teeth (IP P2), the average decrease of plaque index was $95.22 \%$. On IP B statistical test of IP P2 for $0.05 \%$ sodium fluoride mouth wash, the average of plaque index decrease was also significant, this was $93.90 \%$.

$0.2 \%$ chlorhexidine mouth wash used as a positive control, significantly decreases the plaque index, on IP A with IP B, IP A with IP P1 and IP B with IP P1, each with the average value of decreasing plaque index of $17.53 \%, 96.11 \%$ and $95.05 \%$ respectively. Meanwhile, on the test of negative control mouth was (aquadest), there was no change in plaque index on IPA test with IP B $(0 \%)$, even though there was a significant difference in IP A with IP P3 test, and IP B with IP P3 test, where each has the same value of $69.67 \%$.

The intergroup statistical analysis which compared the decrease of plaque index between $0.05 \%$ sodium fluoride mouthwash with $0.2 \%$ chlorhexidine mouthwash, $0.05 \%$ sodium fluoride with Aquadest and $0.2 \%$ chlorhexidine with Aquadest against 3 research time (IP A, IP B and IP $P)$, is shown in Table 4. The average result of table 4 statistically processed to see the significance on table 5. On Table 5, the three tested mouth wash against IP A vs. IP B, IP A vs. IP P and IP B vs. IP P had a significant difference statistically.

Table 5 showed a significant difference between $0.2 \%$ chlorhexidine mouthwash and $0.05 \%$ sodium fluoride on IP Avs. IP B test by $8.40 \%$, which showed that the function of $0.2 \%$ chlorhexidine as a positive control had more influence in reducing the plaque index. The effect of $0.2 \%$ chlorhexidine mouthwash in decreasing the plaque index compared to $0.5 \%$ Sodium Fluoride was also shown in IP A vs. IP P test with IP B vs. IP P test, which was $4.08 \%$ and $5.32 \%$, respectively. 
Table 4. Likeness test on plaque index between solutions

\begin{tabular}{clc}
\hline Mouthwash & Plaque index & Average(\%) \\
\hline IP A vs IP B & CHX vs NaF & 0.26 \\
& $\mathrm{CHX}$ vs $\mathrm{H}_{2} \mathrm{O}$ & 0.44 \\
& $\mathrm{NaF}$ vs $\mathrm{H}_{2} \mathrm{O}$ & 0.41 \\
IP A vs IP P & $\mathrm{CHX}$ vs NaF & 0.42 \\
& $\mathrm{CHX} \mathrm{vs} \mathrm{H}_{2} \mathrm{O}$ & 0.87 \\
& $\mathrm{NaF}$ vs $\mathrm{H}_{2} \mathrm{O}$ & 0.87 \\
IP Bvs IP P & $\mathrm{CHX}$ vs NaF & 0.35 \\
& $\mathrm{CHX}$ vs $\mathrm{H}_{2} \mathrm{O}$ & 0.52 \\
& $\mathrm{NaF}$ vs $\mathrm{H}_{2} \mathrm{O}$ & 0.60 \\
\hline
\end{tabular}

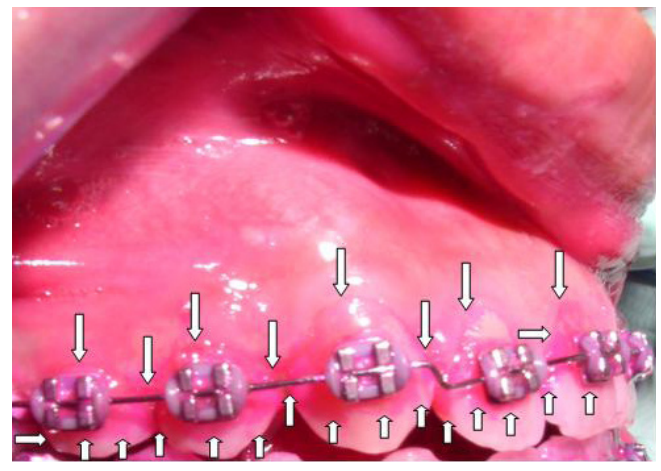

Figure 2. Plaques on maxillary tooth colored with disclosing solution (arrows)

Table 5. Likeness test on plaque index between solution (in \%)

\begin{tabular}{|c|c|c|c|c|c|c|c|}
\hline Plaque Index & Plaque Index & Average (\%) & std & $\mathrm{n}$ & t count & $\mathrm{t}$ table & Characteristic \\
\hline IP A vs IP B & $\begin{array}{l}\mathrm{CHX} \text { vs } \mathrm{NaF} \\
\mathrm{CHX} \text { vs } \mathrm{H} 2 \mathrm{O} \\
\mathrm{NaF} \text { vs } \mathrm{H} 2 \mathrm{O}\end{array}$ & $\begin{array}{c}8.40 \\
17.53 \\
16.45\end{array}$ & $\begin{array}{c}8.522 \\
12.429 \\
14.361\end{array}$ & $\begin{array}{l}16 \\
16 \\
16\end{array}$ & $\begin{array}{l}3.94 \\
5.64 \\
4.58\end{array}$ & $\begin{array}{l}2.13 \\
2.13 \\
2.13\end{array}$ & $\begin{array}{l}\text { Sign } \\
\text { Sign } \\
\text { Sign }\end{array}$ \\
\hline IP A vs IP P & $\begin{array}{l}\mathrm{CHX} \text { vs } \mathrm{NaF} \\
\mathrm{CHX} \text { vs } \mathrm{H} 2 \mathrm{O} \\
\mathrm{NaF} \text { vs } \mathrm{H} 2 \mathrm{O}\end{array}$ & $\begin{array}{c}4.08 \\
26.44 \\
25.55\end{array}$ & $\begin{array}{c}2.425 \\
10.436 \\
10.692\end{array}$ & $\begin{array}{l}16 \\
16 \\
16\end{array}$ & $\begin{array}{c}6.73 \\
10.13 \\
9.56\end{array}$ & $\begin{array}{l}2.13 \\
2.13 \\
2.13\end{array}$ & $\begin{array}{l}\text { Sign } \\
\text { Sign } \\
\text { Sign }\end{array}$ \\
\hline IPA vs IP P & $\begin{array}{l}\mathrm{CHX} \text { vs } \mathrm{NaF} \\
\mathrm{CHX} \text { vs } \mathrm{H} 2 \mathrm{O} \\
\mathrm{NaF} \text { vs } \mathrm{H} 2 \mathrm{O}\end{array}$ & $\begin{array}{c}5.32 \\
25.39 \\
24.23\end{array}$ & $\begin{array}{c}3.351 \\
10.457 \\
10.955\end{array}$ & $\begin{array}{l}16 \\
16 \\
16\end{array}$ & $\begin{array}{l}6.35 \\
9.71 \\
8.85\end{array}$ & $\begin{array}{l}2.13 \\
2.13 \\
2.13\end{array}$ & $\begin{array}{l}\text { Sign } \\
\text { Sign } \\
\text { Sign }\end{array}$ \\
\hline
\end{tabular}

The significant difference was also shown in the changes of IPA vs. IP B on $0.05 \%$ sodium fluoride mouthwash with aquadest, which was $16.45 \%$. The significant change of IP A vs. IP P between $0.05 \%$ sodium fluoride mouthwash and aquadest was $25.55 \%$. The significant change IP B vs. IP P between the two mouthwashes was also shown in Table 5, which was $24.23 \%$. This showed that $0.05 \%$ sodium fluoride mouthwash had more effect in plaque control compared to aquadest as a negative control.

The $0.2 \%$ chlorhexidine mouthwash as a positive control significantly had more influence in decreasing the plaque index compared to aquadest as a negative control. On IP A vs. IP B test of $0.2 \%$ chlorhexidine mouthwash against Aquadest there was a significant difference of $17.53 \%$. On IP A vs. IP P test of $0.2 \%$ chlorhexidine mouth wash against aquadest there was a difference average of $26.44 \%$ and on IP B vs. IP P test both of the mouthwash had an average difference of $25.39 \%$.

\section{DISCUSSION}

The results of this research showed that $0.05 \%$ sodium fluoride mouthwash had an effect in reducing the plaque index, on using together or without additional tooth brushing. This was proven by the statistical results, which showed the significance in the comparison of early plaque index after 24 hours of not brushing the teeth, with the plaque index after rinsing without tooth brushing, and plaque index after 1 week of tooth brushing plus rinsing the mouth with $0.05 \%$ sodium fluoride. These results were also in accordance with Boyd and Chun ${ }^{7}$, who stated that the mouthwash with fluoride can prevent plaque forming. Boyd and Chun $^{7}$ also stated that the use of fluoride mouthwash can be effective to prevent plaque, if used together with other mechanical procedure of plaque removal especially in orthodontic patients, and patients with crowding teeth.

These results also prove that the use of $0,05 \%$ sodium fluoride mouthwash can decrease the plaque index on fixed orthodontics patients [and also recommend the use of mouthwash with fluoride during orthodontics treatments].6,9,10,12,15 Also, McGlynn ${ }^{13}$ proved that the use of $0.05 \%$ sodium fluoride was effective enough to prevent caries and plaque on children patients with orthodontics band. In this research, $0.02 \%$ chlorhexidine mouthwash was proven to decrease the plaque 
index significantly, without tooth brushing (IP A vs. IP B), and by using it for a week with tooth brushing (IPA vs. IP P1 and IP B vs. IP P1). This was in accordance with numerous investigations which stated that $0.2 \%$ chlorhexidine was more effective in reducing plaque. ${ }^{5} 0.05 \%$ sodium fluoride mouthwash compared to $0.2 \%$ chlorhexidine as a positive control showed less influence on plaque control. Comparison of the result on early plaque index, the plaque index after rinsing with mouthwash without brushing the teeth (IPA vs. IP B) was $17.53 \%$ on $0.2 \%$ chlorhexidine, while with $0.05 \%$ sodium fluoride was $16.45 \%$.

The measurement results on plaque index reduction compared to the plaque index 1 week after brushing the teeth and rinsing with the $0.2 \%$ chlorhexidine mouthwash (IP A vs. IP P) was higher $(96.11 \%)$ compared to the $0.05 \%$ sodium fluoride $(95.22 \%)$. The results on samples measurement on plaque index who rinsed using the mouthwash without tooth brushing, compared to samples 1 week after brushing the teeth and rinsing with the mouthwash (IP B vs. IP P) on $0.2 \%$ chlorhexidine also showed higher value (95.05\%) compared to the $0.05 \%$ sodium fluoride (93.90\%). The statistical test on Table 4.5 also showed that the $0.2 \%$ chlorhexidine mouthwash has a significant influence on the decrease of plaque index compared to $0.05 \%$ sodium fluoride on the whole comparison on plaque index. Thus proved that $0.2 \%$ chlorhexidine is still the most influential mouthwash in reducing plaque index on orthodontics patients. ${ }^{16}$

The sodium fluoride mouthwash of $0.05 \%$ concentration compared to the chlorhexidine of $0.2 \%$ concentration was less influential in reducing the plaque index in this research, which also was in accordance with Grant et.al. ${ }^{8}$ who stated that the fluoride concentration less than $0.6 \%$ doesn't have the same capacity with $0.2 \%$ chlorhexidine gluconate in reducing plaque and gingivitis. This also in accordance with Grant et.al. ${ }^{8}$ that the Sodium fluoride mouthwash in 128$2048 \mu \mathrm{g} / \mathrm{ml}( \pm 0.0128-0.2048 \%)$ concentration, has minimal antimicrobial effect and low potential as antibacterial agent.

Plaque index reduce with the use of only $0.05 \%$ sodium fluoride mouthwash will not show optimal result (only 16.45\%) compared to the decrease of plaque index after tooth brushing using $05 \%$ sodium fluoride mouthwash for 1 week. The decrease in plaque index after 24 hours of not brushing the teeth with the plaque index after tooth brushing and using $0.05 \%$ sodium fluoride mouthwash for 1 week (IP A vs. IP P2) showed an optimal result of $95.22 \%$. Meanwhile the plaque index decrease on usage of mouthwash without tooth brushing with plaque index after tooth brushing and using the $0.05 \%$ sodium fluoride mouthwash for 1 week (IP B vs. IP P2) also showed an optimal result of $93.90 \%$. This showed that the use of $0.05 \%$ sodium fluoride mouthwash will only be optimal in decreasing plaque index, if accompanied by tooth brushing.

The decrease of plaque index by using only $0.2 \%$ chlorhexidine mouthwash also did not show an optimal result (only about $17.53 \%$ ). The results proved that $0.2 \%$ chlorhexidine mouthwash has the same effect as $0.05 \%$ sodium fluoride, which will also decrease the plaque index if followed by tooth brushing. The decrease of the plaque index on the comparison of plaque index after 24 hours of non tooth brushing with the plaque index after tooth brushing and using $0.2 \%$ chlorhexidine for one week (IP A vs. IP P1) showed an optimal result of 96.11\%. Meanwhile, the plaque index comparison on using the mouthwash without tooth brushing, with the plaque index after tooth brushing and using the $0.2 \%$ chlorhexidine mouthwash for 1 week (IP B vs. IP P1) also showed optimal result of $95.05 \%$. These results are in accordance with the other investigations about the function of mouthwash as assistance in plaque control, and not as a replacement of plaque removal by mechanical means (i.e. tooth brushing). ${ }^{5}$

The comparison on reduction of plaque index on $0.05 \%$ sodium fluoride mouthwash compared to Aquadest as a negative control showed a significant difference (table 4 and table 5). There was no decrease in plaque index between early plaque indexes on samples without tooth brushing for 24 hours, with the plaque index on samples rinsing with aquadest without tooth brushing (IP A vs. IP $B)$. The reduction on plaque index happened after samples were instructed to brush their teeth and rinse with aquadest provided for 1 week, which was $69.67 \%$. This showed that aquadest has no effect on the reduction of plaque index. The result of this investigation is supporting to the results from Chaves ${ }^{3}$ and Newman ${ }^{17}$, who stated that even 
though water can be used as irrigation after tooth brushing, the water itself cannot reduce formed plaque chemically. The reduction on plaque index with the use of aquadest in this investigation, happened after samples brushed their teeth and used aquadest for 1 week (IP P3), this proved that the reduction of plaque index happened because of tooth brushing and not the use of aquadest.

\section{CONCLUSION}

The result of this research showed that there was a significant reduction in plaque index $(p=0.05)$ using $0.05 \%$ sodium fluoride mouthwash, compared to aquadest, but chlorhexidine mouthwash was proven to have the biggest effect than $0,05 \%$ sodium fluoride on reducing plaque index. Also, the result of this research showed that on every mouthwash tested, there was a significant reduction on plaque index after the samples brushed their teeth and rinse using the mouthwash. This showed that plaque control by mechanical means (tooth brushing) is always the most influential way in reducing plaque on fixed orthodontic patients. Mouthwash is still the chemical assistance in reducing plaque.

\section{REFERENCES}

1. Stanley RN. Orthodontic diagnosis and treatment planning. In: Bishara SE, editor. Textbook of orthodontics. Philadelphia: WB Saunders Co; 2001.

2. Kilicoglu H, Yildirim M, Polater H. Comparison of the effectiveness of two types of toothbrushes on the oral hygiene of patients undergoing orthodontic treatment with fixed appliances. Am J Orthod Dentofac Orthop 1997;111:5914.

3. Clark JR. Oral hygiene in the orthodontic practice: motivation, responsibilities and concepts. Am J Orthod Dentofac Orthop 1976;69:72-82.

4. Chung A, Kudlick EM, Gregory JE, Royal GC, Reindorf CA. Toothbrushing and transient bacteremia in patients undergoing orthodontic treatment. Am J Orthod Dentofac Orthop 1986;90:181-6.

5. Carranza FA, Newman MG. Clinical periodontology: the gingiva; the tooth- supporting structures; orthodontic considerations in periodontal therapy. $8^{\text {th }}$ ed. Philadelphia: WB. Saunders Co;1996.

6. Hobson RS, Clark JD. How UK orthodontist advise patiens to oral hygiene. $\mathrm{Br} \mathrm{J}$ Orthod 1998;25:64-6.

7. Boyd RL, Chun YS. Eighteen-month evaluation of the effect of a 0.4 percent stannous fluoride gel on gingivitis in orthodontic patients. Am J Orthod Dentofac Orthop 1994;105:35-41.

8. Grant A, Stern I, Lisgarten MA. Periodontics: sensitivity of periodontal organisms to antibiotics and other antimicrobial agents. $6^{\text {th }}$ ed. St Louis: Mosby Co; 1988.

9. Zachrisson BU. Periodontal condition associated with orthodontic treatment. II. histologic findings. The Angle Orthodontist 1973;43(4):352-7.

10. McGlynn FD, LeCompte EJ, Thomas RG, Courts FJ, Melamed BG. Effects of behavioral selfmanagement on oral hygiene adherence among orthodontic patients. Am J Orthod Dentofac Orthop 1987;91: 5-21.

11. Boyd RL, Murray P, Robertson PB. Effect of rotary electric toothbrush versus manual toothbrush on periodontal status during orthodontic treatment. Am J Orthod Dentofac Orthop 1989;96:342-7.

12. Hind V. Orthodontic products update fluoride mouthrinses. Br J Orthod 1999;26:242-3.

13. Chaves ES, Kornman KS, Manwell MA, Jones AA, Nebold DA, et al. Mechanism of irrigation effects on gingivitis. J Periodontol 1994;65: 1016-21.

14. Lindhe J. Textbook of clinical periodontology: epidemiology of periodontal disease, pathogenesis of plaque-associated periodontal disease. $2^{\text {nd }}$ ed. Munksgaard: Blackwell 2003.

15. Boyd RL. Comparison of three self-applied topical fluoride preparations for control of decalcification. The Angle Orthodontist 1993; 63:25-30.

16. Yeung SCM, Howell S, Fahey P. Oral hygiene program for orthodontic patients. Am J Orthod Dentofac Orthop 1989;96:208-13.

17. Newman MG, Flemming TF, Nachnani S, Rodrigues A, Calsina G, Lee YS, et al. Effectiveness of adjunctive irrigation in early periodontitis: Multi-center evaluation. J Periodontol 1994;65:224-9. 